\title{
Comparison of the Transition Dipole Moments Calculated by TDDFT with High Level Wavefunction Theory
}

David Robinson*

Department of Chemistry and Forensics, School of Science and Technology, Nottingham Trent University, Clifton Lane, Nottingham, NG11 8NS, United Kingdom

*david.robinson@ntu.ac.uk

\begin{abstract}
Time dependent density functional theory (TDDFT) is widely used to simulate the excited states of organic and inorganic molecules. We calculate the transition dipole moments (TDM) for a selection of commonly employed exchange-correlation functionals for a test set of 15 molecules and compare them with both EOM-CC3 and ADC(3) calculated TDMs, which we use as a benchmark. Contemporary range-separated hybrid functionals perform the best for both direction and magnitude, while "pure" local hybrids should be employed with caution.
\end{abstract}




\section{Introduction}

Time-dependent density functional theory (TDDFT) has seen widespread use for the calculation of excited-state transition energies and properties, due to algorithmic advances and the vast increases in computer speeds in the past two decades. TDDFT has been applied to a wide variety of fields, including biomolecular probes, molecular assemblies and photochemical reactions. In general, most researchers use the adiabatic approximation when applying TDDFT, changing the exchange-correlation functional and/or basis set to achieve a desired level of accuracy. Often, the choice of exchange correlation functional is based on some comparison of transition energies with experiment or calibration against higher-level wavefunction theories, ${ }^{1-}$ ${ }^{21}$ see the recent review by Laurent and Jacquemin for a full discussion. ${ }^{22}$ Other authors have suggested state-specific properties as an indication of the quality of the description of the excited state by TDDFT. Tozer et $\mathrm{al}^{23}$ developed a diagnostic value to determine the electronic nature of a given transition (e.g. charge-transfer, Rydberg etc.), while Jacquemin ${ }^{24}$ investigated the excited state dipole and traceless quadrupole moment for a range of molecules, concluding that there is a relatively limited dependency of these properties on the choice of exchange correlation functional, except when charge-transfer states are involved.

Other groups have looked at specific transition properties, namely the oscillator strength, $f$, of the transition. ${ }^{25-27}$ Tawada et al. ${ }^{25}$ found that the range-separated hybrid (RSH) functionals reproduced reference wavefunction oscillator strengths (SAC-CI) and experimental trends better than other types of functional. Timzeghazin et al. ${ }^{26}$ found that TDDFT in general overestimates oscillator strengths, while Caricato et al. ${ }^{27}$ performed a wide-ranging study, concluding that CAM-B3LYP (an RSH functional) performed the best overall. The oscillator strength for a transition between states $M$ and $N$ is given by

$f_{M N}=\frac{2}{3} \Delta E\left\langle N\left|\mu_{M N}\right| M\right\rangle$ 
where $\left\langle N\left|\mu_{M N}\right| M\right\rangle$ is the transition dipole moment (TDM) between states $M$ and $N$. The TDM is a key feature of any excited state method and is integral to the correct qualitative and quantitative description of a given transition. Any method that correctly describes the ground state and excited state electronic densities should give accurate transition dipole moments. The direction of the TDM vectors are commonly used to determine the nature (and symmetry, where appropriate) of a transition. For example, in aromatic molecules, Platt's notation ${ }^{28}$ is commonly used. For benzene, the ${ }^{1} \mathrm{~L}_{\mathrm{a}}\left({ }^{1} \mathrm{~B}_{2 \mathrm{u}}\right),{ }^{1} \mathrm{~L}_{\mathrm{b}}\left({ }^{1} \mathrm{~B}_{1 \mathrm{u}}\right)$ and ${ }^{1} \mathrm{~B}\left({ }^{1} \mathrm{E}_{1 \mathrm{u}}\right)$ transitions are the lowlying valence $\pi \rightarrow \pi^{*}$ excitations. The transition dipole moment vectors between the ${ }^{1} \mathrm{~L}_{\mathrm{a}}$ and ${ }^{1} \mathrm{~L}_{\mathrm{b}}$ transitions are at an angle of $\sim 90^{\circ}$ to each other. This rule is often applied to pseudosymmetric aromatic molecules of biochemical interest, such as tryptophan and it's derivatives. ${ }^{29-31}$ Conformational dependence of the TDM direction has also been observed, where subtle changes in the molecular structure of the molecules can result in large changes in the TDM direction. ${ }^{32}$ Therefore, it is of the upmost importance that a selected functional employed for a TDDFT calculation can correctly reproduce the TDM for a given transition, to aid in identifying the transition and to quantitatively predict the oscillator strength, $f$.

In the current study, we compare both the direction and magnitude of the transition dipole moment with EOM-CC3 for a range of molecules and selection of exchange-correlation functionals for singlet vertical excitations.

\section{Computational Details}

Ground state geometries of the molecules given in Figure 1 were optimised using the B3LYP functional $^{33}$ (as implemented in the Q-Chem 5.0 package) and 6-31G(d) basis set. ${ }^{34,35}$ Vibrational frequency calculations were performed to ensure the stationary points found were 
true minima. The optimised geometries (in their standard orientation) were used as the basis for the excited state calculations. These Cartesian coordinates are given in the Supporting Information. These molecules were chosen as various combinations of them have appeared in previous benchmarking studies of excited state energies and properties with TDDFT.

Equation of motion iterative coupled cluster with connected triples $(\text { EOM-CC } 3)^{36,37}$ calculations were performed for molecules $\mathbf{1}$ - $\mathbf{1 0}$ using the 6-31+G(d) basis set, solving for the first two singlet excited states in each irreducible representation for each molecule. This method was been chosen as, in previous studies, it was proven to give very accurate transition energies, excited state geometries and oscillator strengths. ${ }^{38-43}$ The two lowest energy transitions with non-zero TDMs were selected as the " $\mathrm{S}_{1}$ " and " $\mathrm{S}_{2}$ " transitions used in this work. Third-order algebraic diagrammatic construction $(\mathrm{ADC}(3))^{44,45}$ calculations were performed for each of the molecules (with the same basis set); for molecules $\mathbf{1}-\mathbf{1 0}$, we benchmarked them against the EOM-CC3 calculated TDMs. For molecules $\mathbf{1 1}-\mathbf{1 5}$, which are too large to apply the EOM-CC3 method, we used $\mathrm{ADC}(3)$ calculations as the benchmark. TDDFT calculations were performed for each molecule using the following functionals: BLYP, ${ }^{46,47}$ BP86, ${ }^{46,48}$ M06-L, ${ }^{49}$ M11-L,${ }^{50}$ OLYP,${ }^{47,51}$ B3LYP, ${ }^{33}$ PBE0, ${ }^{52}$ M06, ${ }^{53}$ BMK, ${ }^{54}$ M06-2X ${ }^{53}$ CAM-B3LYP, ${ }^{55}$ M11, ${ }^{56} \omega \mathrm{B} 97 \mathrm{X}^{57}$ and $\omega \mathrm{B} 97 \mathrm{X}-\mathrm{D} .{ }^{58}$ The first five functionals are "pure" exchange-correlation functionals (no exact exchange), the next five functionals are global hybrid functionals (arranged in increasing order of exact exchange), while the last four are range-separated hybrid (RSH) functionals. The $\omega$ B97X and $\omega$ B97X-D functionals are related to one another; the latter has an empirical correction for dispersion interactions, which doesn't explicitly contribute to the excited state calculation, but rather indirectly contributes through a re-parameterisation of the functional. TDDFT calculations were performed both with and without the Tamm-Dancoff approximation (TDA) $)^{59}$ (denoted TDDFT and TDDFT/TDA, respectively) and the $6-31+G(d)$ basis set; for all other options, the default with the Q-Chem 
$5.0^{60}$ electronic structure program were used. Only singlet vertical excitations were calculated, including the first five excited states. The EOM-CC3 calculations were performed with the CFOUR software suite. ${ }^{61}$ 


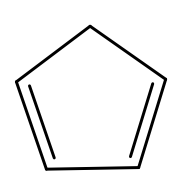

1

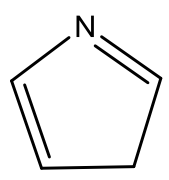

2

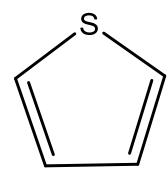

3

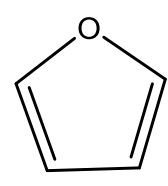

4<smiles>Oc1ccccc1</smiles>

5<smiles>O=C(O)c1ccccc1</smiles>

6<smiles>CC=CC=CC</smiles>

7<smiles>c1ccc2ccccc2c1</smiles>

8<smiles>O/C=C/C(O)=C/CO</smiles>

9<smiles>c1ccc2[nH]ccc2c1</smiles>

10<smiles>COc1ccc2ccc(=O)oc2c1</smiles>

11<smiles>c1ccc2cc3ccccc3cc2c1</smiles>

14<smiles>Nc1ccc2c(c1)C(=O)CC2=O</smiles>

12<smiles>Nc1ccc(N)c2c1C(=O)C=CC2=O</smiles>

13

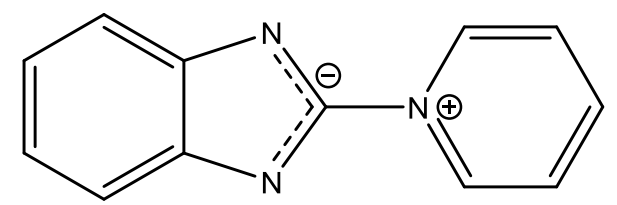

15

Figure 1. Molecular structures of the molecules considered in this work. 


\section{Results and Discussion}

The vertical transition energies and oscillator strengths calculated by the various methods are not the focus of the current work, but are given in the Supporting Information for completeness.

\subsection{Transition dipole moment direction}

The average angle of deviation between each of the TDDFT/TDA TDMs, and the EOM-CC3 TDM for the different functionals are given in Table 1, along with the deviation between the ADC(3) and EOM-CC3 TDMs for molecules 1 - 10. ADC(3) calculated TDMs compare well with the EOM-CC3 TDMs, especially for transitions with $f>1 \times 10^{-3}$, with average errors in the angles for $S_{1}$ and $S_{2}$ TDMs lower than the corresponding TDDFT/TDA values when considering both transitions. The magnitude of the ADC(3) TDMs also compares favourably (see later; section 3.2). This gives us confidence that the $\mathrm{ADC}(3)$ method is suitable as a benchmark for the molecules that are too large to apply the EOM-CC3 method. The average angle of deviation from the EOM-CC3 TDM for the first two valence singlet excited states for all functionals is less than 13 degrees, although the maximum deviations are considerably higher (see below). Given in Figures 2 and 3 are the maximum angles of deviation from the EOM-CC3 TDM when only transitions with an oscillator strength greater than $1 \times 10^{-3}$ are included, for $S_{1}$ and $S_{2}$ transitions, respectively. While this is a somewhat arbitrary choice for the oscillator strength, it highlights that many of the large deviations in TDM angles are found for weak transitions, where the overall magnitude of the TDM is small and so the direction of the TDM is often less important. This is especially true for the $\mathrm{S}_{2}$ transitions (Figure 3), where the maximum deviation is reduced for all functionals. Large reductions in the errors are observed for the $S_{1}$ transitions for the M06-2X, CAM-B3LYP and $\omega$ B97X-D functionals, with comparatively smaller reductions in the angles of deviation for the BLYP, OLYP and M11 
functionals. The deviations calculated by the other functionals remain unchanged, indicating TDMs of larger magnitude deviate from the EOM-CC3 TDM.

For the $\mathrm{S}_{1} \mathrm{TDMs}$, the average and maximum angles of deviation decrease with increasing amounts of exact exchange. The "pure" density functionals, BLYP, BP86, M06-L and OLYP, all have average angles of deviation greater than $9^{\circ}$, while the average deviation decreases from $12.9^{\circ}$ to $6.5^{\circ}$ with increasing proportions of exact exchange in the hybrid functionals (values given for PBE0 and M06-2X, respectively). This is not surprising, since charge-transfer states are involved for a few of the molecules considered for which standard functionals are known to fare poorly. ${ }^{62}$ The RSH functionals M11 and $\omega$ B97X have average angles of deviation of less than $6^{\circ}$, with M11 performing the best for this class of functional. The maximum angles of deviation (excluding transitions where $f<0.001$ ) show a similar pattern, with the RSH functionals and M06-2X all having maximum values of less than $15^{\circ}$. The average angles of deviation for the $S_{2}$ transitions (Table 2) follow a similar trend to those observed for the $S_{1}$ transitions, although the maximum deviations are higher for most methods compared to those seen for the $S_{1}$ transitions (Figure 3).

For molecules 11 - 15, where ADC(3) TDMs are used as a benchmark, a similar picture emerges (Figures 4 and 5; Table 2); the RSH functionals again compare the best with the reference TDMs for the $\mathrm{S}_{1}$ transitions, while M06 has the lowest average angle of deviation for the $S_{2}$ transitions. When only the transitions with an oscillator strength greater than $1 \times 10^{-3}$ are considered, the RSH functional M11 performs the best (Figures 4 and 5), while the hybrid functionals B3LYP, PBE0, M06 and BMK have the highest deviations for the $\mathrm{S}_{2}$ transitions. 


\begin{tabular}{|c|c|c|c|c|}
\hline Functional & $\left\langle\theta_{S_{1}}\right\rangle /$ degrees & $\left\langle\theta_{S_{2}}\right\rangle /$ degrees & $\theta_{S_{1}}^{\max }$ & $\theta_{S_{2}}^{\max }$ \\
\hline BLYP & 9.1 & 8.2 & 27.0 & 42.6 \\
\hline BP86 & 10.0 & 5.5 & 25.9 & 26.8 \\
\hline M06-L & 9.7 & 6.8 & 34.0 & 39.1 \\
\hline M11-L & 7.3 & 6.3 & 21.9 & 29.5 \\
\hline OLYP & 9.1 & 5.3 & 27.5 & 24.1 \\
\hline B3LYP & 12.0 & 7.5 & 45.2 & 42.4 \\
\hline PBE0 & 12.9 & 7.1 & 55.3 & 39.0 \\
\hline M06 & 9.4 & 8.4 & 42.5 & 46.7 \\
\hline BMK & 8.9 & 5.2 & 31.5 & 22.1 \\
\hline M06-2X & 6.5 & 6.9 & 24.4 & 40.5 \\
\hline CAM-B3LYP & 8.4 & 7.3 & 34.1 & 39.2 \\
\hline M11 & 2.9 & 7.1 & 13.1 & 43.9 \\
\hline$\omega B 97 X$ & 5.2 & 6.2 & 16.9 & 29.2 \\
\hline$\omega B 97 X-D$ & 9.9 & 6.2 & 56.3 & 28.6 \\
\hline $\operatorname{ADC}(3)$ & 4.8 & 6.4 & 25.5 & 38.7 \\
\hline
\end{tabular}

Table 1. Average angle of deviation of the TDDFT/TDA TDMs from the CC3 TDM for molecules 1 - $\mathbf{1 0}$. 


\begin{tabular}{lcccc}
\hline Functional & $\left\langle\theta_{S_{1}}\right\rangle /$ degrees & $\left\langle\theta_{S_{2}}\right\rangle /$ degrees & $\theta_{S_{1}}^{\max }$ & $\theta_{S_{2}}^{\max }$ \\
\hline BLYP & 15.8 & 15.0 & 74.2 & 61.8 \\
BP86 & 15.8 & 15.0 & 73.8 & 59.3 \\
M06-L & 17.0 & 16.9 & 84.7 & 70.8 \\
M11-L & 15.3 & 14.2 & 75.5 & 58.0 \\
OLYP & 15.9 & 13.8 & 74.1 & 58.3 \\
\hline B3LYP & 12.7 & 7.5 & 61.7 & 19.0 \\
PBE0 & 11.1 & 7.9 & 53.6 & 23.3 \\
M06 & 10.5 & 6.5 & 51.3 & 22.0 \\
BMK & 3.5 & 12.8 & 16.4 & 47.7 \\
M06-2X & 3.4 & 16.4 & 15.6 & 73.0 \\
\hline CAM-B3LYP & 1.9 & 11.0 & 9.0 & 43.7 \\
M11 & 6.1 & 19.6 & 30.3 & 86.8 \\
$\omega$ B97X & 3.3 & 15.2 & 16.5 & 66.2 \\
$\omega$ B97X-D & 2.7 & 10.9 & 12.6 & 41.9 \\
& & & & 4.9 \\
\hline
\end{tabular}

Table 2. Average angle of deviation of the TDDFT/TDA TDMs from the ADC(3) TDM for molecules 11 - 15. 


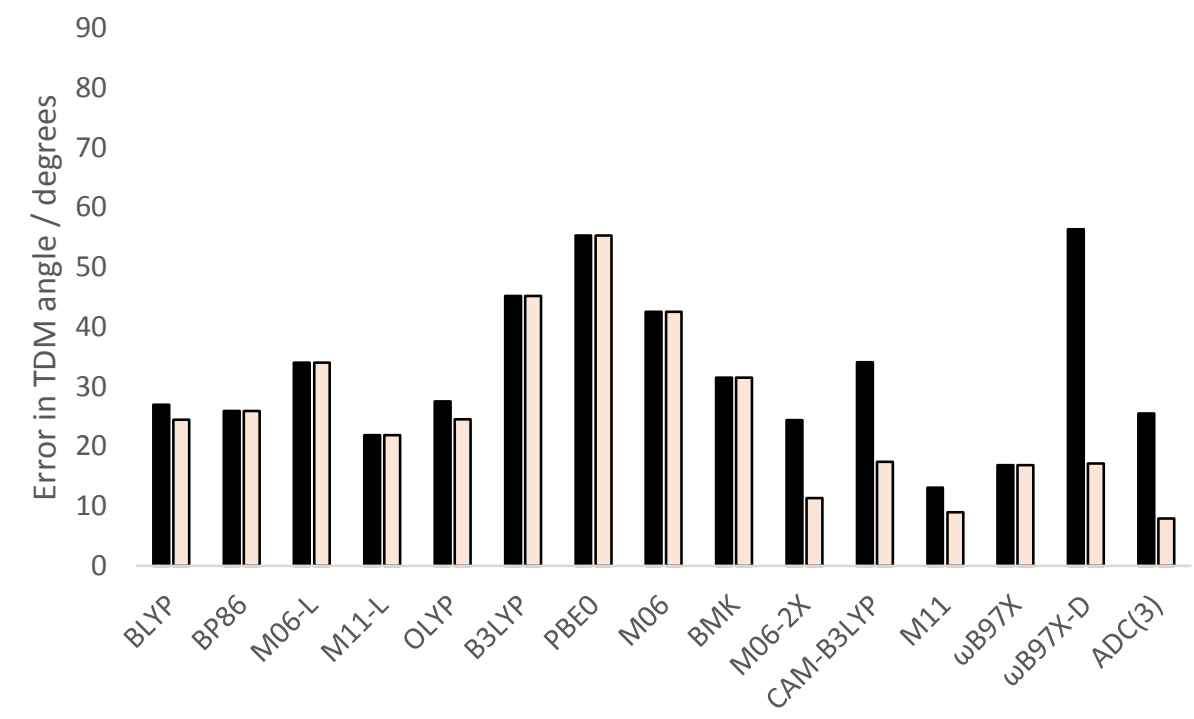

Figure 2. Maximum angle of deviation between the TDDFT/TDA calculated TDM and the CC3 TDM for the $S_{1}$ transitions for molecules 1 - 10: all transitions (filled bars); transitions with $f>0.001$ (open bars).

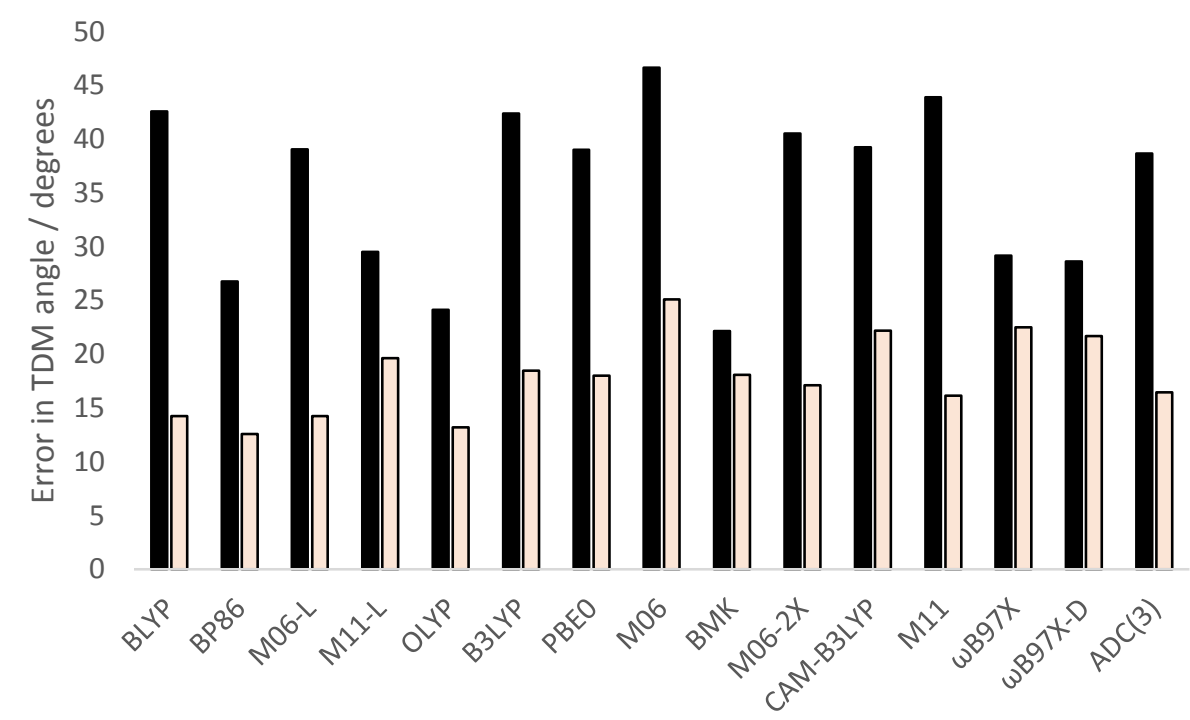

Figure 3. Maximum angle of deviation between the TDDFT/TDA calculated TDM and the CC3 TDM for the $S_{2}$ transitions for molecules 1 - 10: all transitions (filled bars); transitions with $f>0.001$ (open bars). 


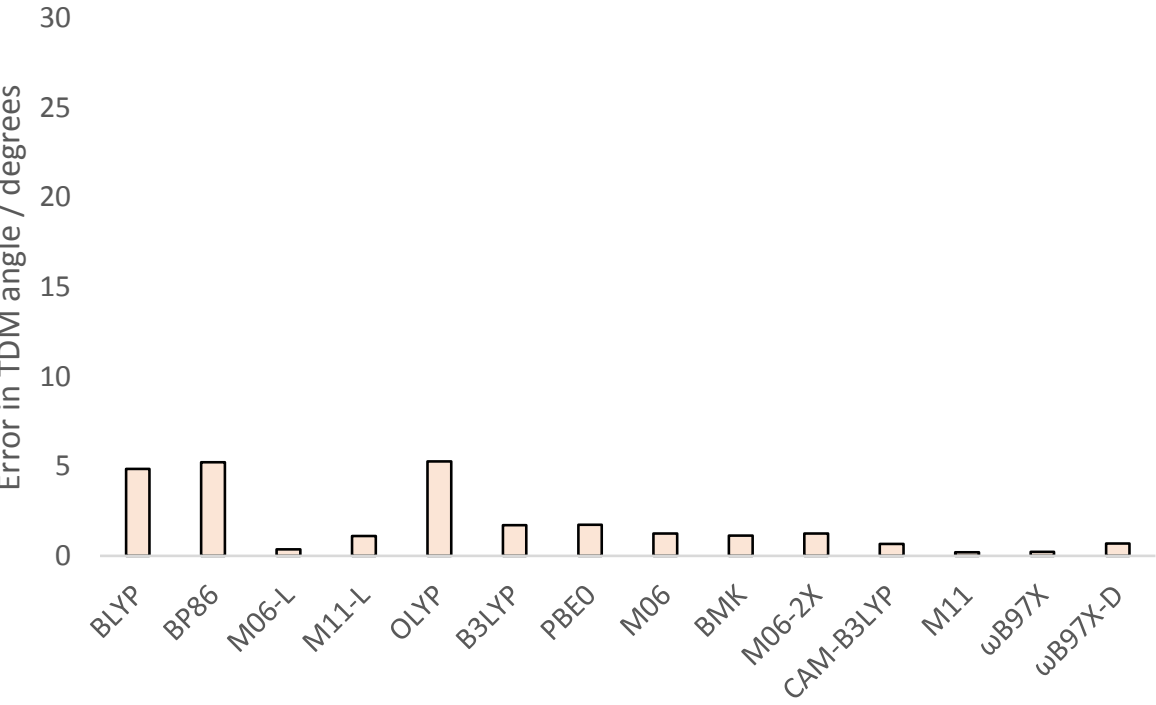

Figure 4. Maximum angle of deviation between the TDDFT/TDA calculated TDM and the $\operatorname{ADC}(3) \mathrm{TDM}$ for the $\mathrm{S}_{1}$ transitions for molecules $\mathbf{1 1}$ - $\mathbf{1 5}$ (only transitions with $f>0.001$ are shown).

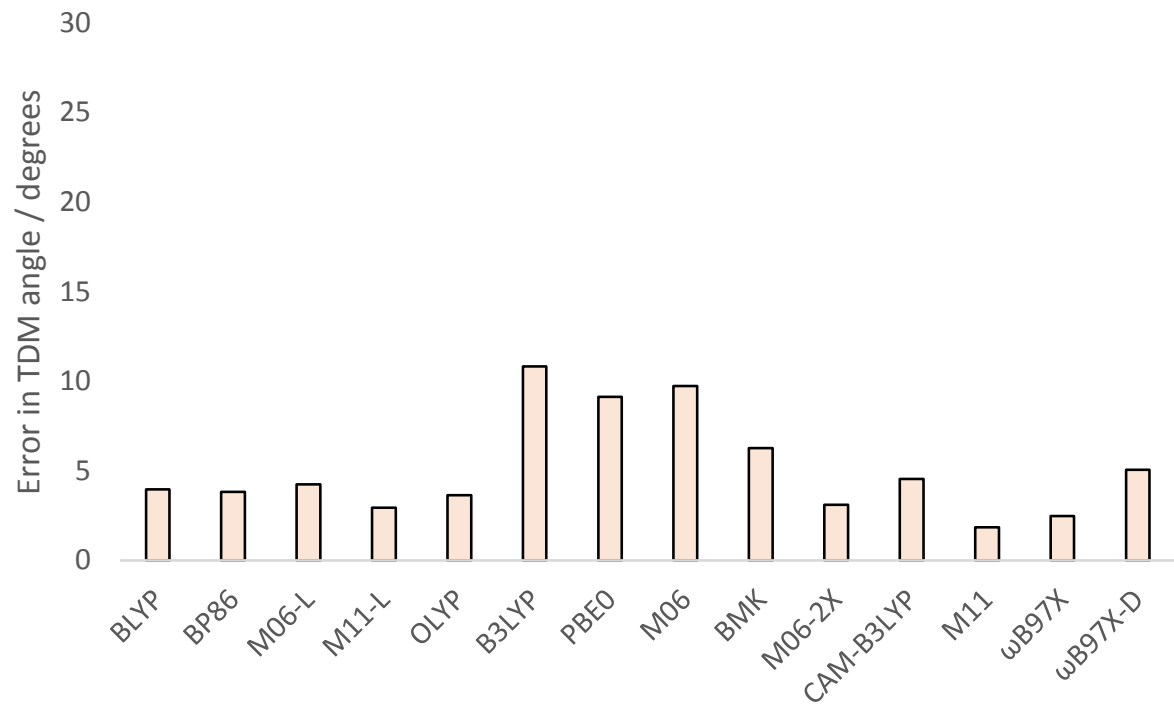

Figure 5. Maximum angle of deviation between the TDDFT/TDA calculated TDM and the ADC(3) TDM for the $\mathrm{S}_{2}$ transitions for molecules $\mathbf{1 1}$ - $\mathbf{1 5}$ (only transitions with $f>0.001$ are shown). 
The average deviation angles given above (Table 1) include molecules which have higher symmetry than $C_{1}$ or $C_{s}$. Some of the molecules in the present study belong to the $C_{2 v}$ or $D_{2 h}$ point groups, in which case the deviation is zero degrees, since the direction of the TDMs are determined by symmetry. Given in Table 3 are the average angles of deviation for those molecules with symmetry belonging to either the $\mathrm{C}_{1}$ or $\mathrm{C}_{\mathrm{s}}$ point groups. As expected, the average angle of deviation increases for each of the methods, as they are not restricted by symmetry. 


\begin{tabular}{lcc}
\hline Functional & $\left\langle\theta_{S_{1}}\right\rangle /$ degrees & $\left\langle\theta_{S_{2}}\right\rangle /$ degrees \\
\hline BLYP & 11.4 & 10.3 \\
BP86 & 12.5 & 6.9 \\
M06-L & 12.1 & 8.5 \\
M11-L & 9.1 & 7.9 \\
OLYP & 11.4 & 6.7 \\
\hline B3LYP & 15.0 & 9.3 \\
PBE0 & 16.1 & 8.8 \\
M06 & 11.8 & 10.5 \\
BMK & 11.1 & 6.6 \\
M06-2X & 8.1 & 8.7 \\
\hline CAM-B3LYP & 10.5 & 9.1 \\
M11 & 3.7 & 8.8 \\
$\omega B 97 X$ & 6.5 & 7.8 \\
$\omega B 97 X-D$ & 12.4 & 7.8 \\
\hline ADC(3) & 6.9 & 9.1 \\
\hline
\end{tabular}

Table 3. Average angle of deviation of the TDDFT/TDA TDM from the CC3 TDM for molecules belonging to $\mathrm{C}_{1}$ or $\mathrm{C}_{\mathrm{s}}$ point groups. 


\subsection{Magnitude of the transition dipole moments}

While the direction of a given TDM is important in identifying the transition, the magnitude of the TDM is necessary to give quantitative accuracy for the oscillator strength (see equation 1). Given in Figures 6 and 7 are the average magnitudes of the TDMs calculated using TDDFT/TDA as a percentage of the magnitude of the EOM-CC3 TDM and ADC(3) TDM, respectively, such that $100 \%$ means the magnitudes are equal between a given functional and the reference TDM. For most of the methods, the average magnitudes of the TDMs are in the range $80-110 \%$ of the EOM-CC3 TDM, apart from the M11 and OLYP functionals. For M06L, the relatively high average error stems from the TDM for the $S_{1}$ transition of molecule 9 being approximately four times that of the reference EOM-CC3 TDM, although this is a weak transition $\left(f<1 \times 10^{-3}\right)$ and such an error is not such an issue in practice.

The ADC(3) method exhibits a consistent performance in comparison to the EOM-CC3 TDMs for molecules $\mathbf{1}-\mathbf{1 0}$, with average magnitudes of the TDM of $90.3 \%$ (values in the range $85.6 \%$ to $94.8 \%$ ) and $102.1 \%$ (values in the range $80.7 \%$ to $130.1 \%$ ) of the EOM-CC3 TDMs for the $S_{1}$ and $S_{2}$ transitions, respectively. The RSH functionals give a consistent performance versus the $A D C(3)$ method for both $S_{1}$ and $S_{2}$ transitions (Figure 7), with values in the range of $85-90 \%$ of the magnitude of the ADC(3) TDMs.

\subsection{Use of TDDFT without the Tamm-Dancoff approximation}

While the default option for some electronic structure software packages when performing TDDFT calculations is to invoke the Tamm-Dancoff approximation, it is useful to consider the performance of full TDDFT against the benchmark methods. Given in Tables S16 and S17, and Figures S1 - S6 (in the Supporting Information) are the data for the full TDDFT approach. Overall, the TDMs calculated by TDDFT have a slightly larger magnitude than those calculated 
with TDDFT/TDA (e.g. compare Figure S3 and Figure 6), although the trends are very similar. The average angles of deviation from the reference EOM-CC3 (Table S16) show an increase for the $S_{1}$ transitions (in comparison to the TDDFT/TDA values), while the majority of the angles of deviation for the $\mathrm{S}_{2}$ transitions show a decrease versus the TDDFT/TDA values. The maximum angles of deviation are often larger for TDDFT than TDDFT/TDA when including all transitions (see Figure S1 and Figure 2), although for transitions with an oscillator strength greater than $1 \times 10^{-3}$, the profiles are very similar (Figures S1, S2, 2 and 3). 


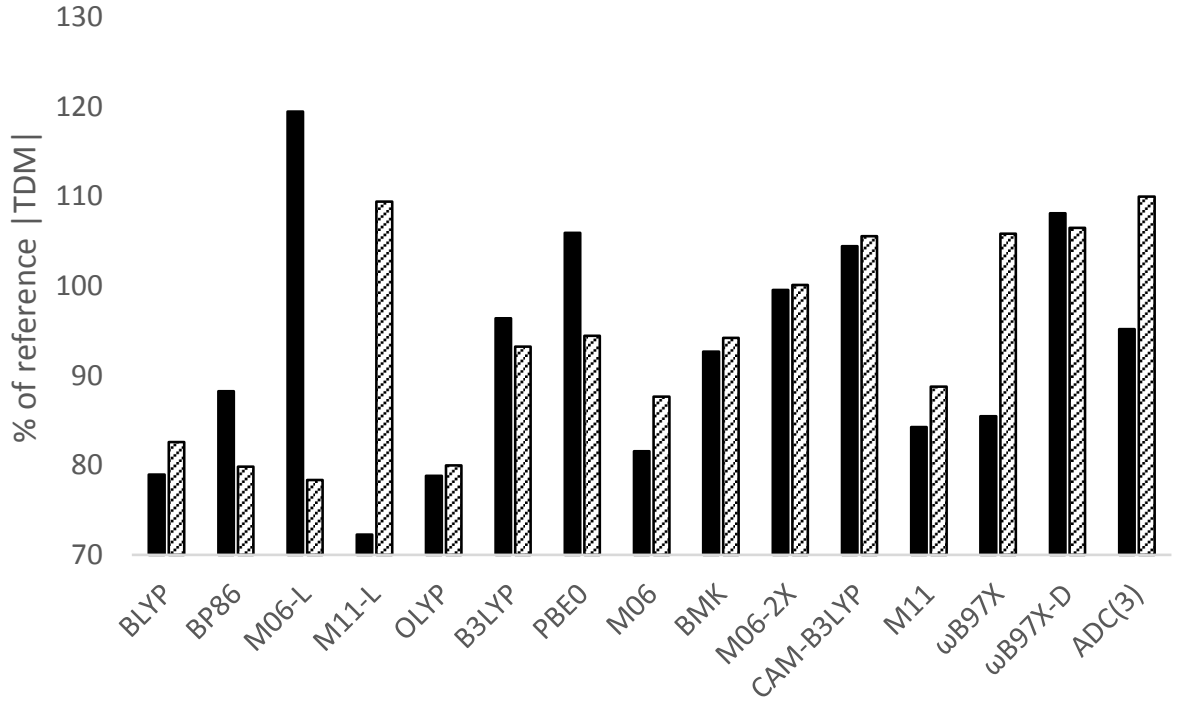

Figure 6. Magnitude of the TDDFT/TDA TDM represented as a percentage of the CC3 TDM for molecules 1 - 10. TDMs with an equal magnitude of the EOM-CC3 TDM have a value of $100 \%$. Note that the $\mathrm{y}$-axis starts at $80 \%$. $\mathrm{S}_{1}$ transitions (filled bars); $\mathrm{S}_{2}$ transitions (patterned bars).

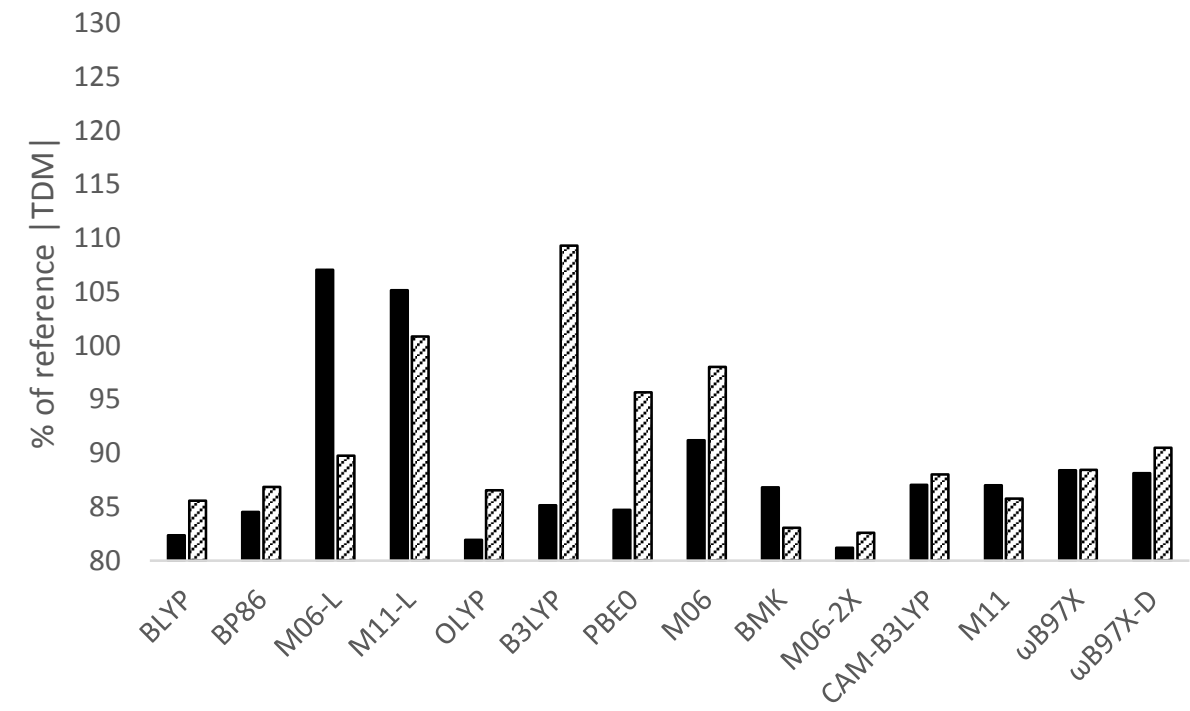

Figure 7. Magnitude of the TDDFT/TDA TDM represented as a percentage of the ADC(3) TDM for molecules 11 - 15. TDMs with an equal magnitude of the EOM-CC3 TDM have a value of $100 \%$. Note that the $y$-axis starts at $80 \% . \mathrm{S}_{1}$ transitions (filled bars); $\mathrm{S}_{2}$ transitions (patterned bars). 


\section{Conclusions}

We have presented data for the direction and magnitude of TDMs calculated using TDDFT both with and without the Tamm-Dancoff approximation and a variety of functionals in comparison with benchmark EOM-CC3 TDMs. In general, the RSH functionals perform well across a wide variety of excitations and molecules, and we recommend choosing one of these. While the $\omega \mathrm{B} 97 \mathrm{X}-\mathrm{D}$ functional has performed slightly worse than the $\omega \mathrm{B} 97 \mathrm{X}$ functional in the current study, we would still recommend this functional where non-covalent interactions are considered important; none of the molecules in this study belong to that class of molecule. Additionally, the M06-2X functional, with 54\% exact exchange incorporated into the functional, also performs well. The local "pure" functionals should be used with caution, although the magnitude of the calculated TDMs for this class of functional compare well with EOM-CC3. The interested reader should therefore carefully select a functional based on the current results and those of other TDDFT benchmarks, to best suit their needs.

\section{Acknowledgements}

DR thanks Nottingham Trent University for provision of time on the Hamilton High Performance Computing facility.

\section{Supporting Information}

Cartesian coordinates of the B3LYP/6-31G(d) optimised geometries; excitation energies and oscillator strengths for all transitions considered. Full data for the full TDDFT transitions. The following files are available free of charge: TDM comparisons - SI.pdf 



\section{References}

1. Hellweg, A.; Gruen, S. A.; Haettig, C. Benchmarking the performance of spin-component scaled CC2 in ground and electronically excited states. Physical Chemistry Chemical Physics 2008, 10, 4119-4127.

2. Henderson, T. M.; Izmaylov, A. F.; Scuseria, G. E.; Savin, A. Assessment of a middlerange hybrid functional. Journal of Chemical Theory and Computation 2008, 4, 12541262.

3. Huenerbein, R.; Grimme, S. Time-dependent density functional study of excimers and exciplexes of organic molecules. Chem. Phys. 2008, 343, 362-371.

4. Huix-Rotllant, M.; Ipatov, A.; Rubio, A.; Casida, M. E. Assessment of dressed timedependent density-functional theory for the low-lying valence states of 28 organic chromophores. Chem. Phys. 2011, 391, 120-129.

5. Iikura, H.; Tsuneda, T.; Yanai, T.; Hirao, K. A long-range correction scheme for generalized-gradient-approximation exchange functionals. J. Chem. Phys. 2001, 115, 3540-3544.

6. Imamura, Y.; Nakai, H. Time-dependent density functional theory (TDDFT) calculations for core-excited states: Assessment of an exchange functional combining the Becke88 and van Leeuwen-Baerends-type functionals. Chemical Physics Letters 2006, 419, 297 303.

7. Improta, R.; Barone, V.; Santoro, F. Ab initio calculations of absorption spectra of large molecules in solution: Coumarin C153. Angewandte Chemie-International Edition 2007, 46, 405-408.

8. Improta, R.; Santoro, F.; Barone, V.; Lami, A. Vibronic Model for the Quantum Dynamical Study of the Competition between Bright and Charge-Transfer Excited States in Single-Strand Polynucleotides: The Adenine Dimer Case. Journal of Physical Chemistry a 2009, 113, 15346-15354.

9. Isegawa, M.; Peverati, R.; Truhlar, D. G. Performance of recent and high-performance approximate density functionals for time-dependent density functional theory calculations of valence and Rydberg electronic transition energies. J. Chem. Phys. 2012, 137, 244104.

10. Jacquemin, D.; Perpete, E. A.; Scalmani, G.; Frisch, M. J.; Ciofini, I.; Adamo, C. Absorption and emission spectra in gas-phase and solution using TD-DFT:

Formaldehyde and benzene as case studies. Chemical Physics Letters 2006, 421, 272276.

11. Jacquemin, D.; Bouhy, M.; Perpete, E. A. Excitation spectra of nitro-diphenylaniline: Accurate time-dependent density functional theory predictions for charge-transfer dyes. J. Chem. Phys. 2006, 124, 204321. 
12. Jacquemin, D.; Preat, J.; Wathelet, V.; Perpete, E. A. Substitution and chemical environment effects on the absorption spectrum of indigo. J. Chem. Phys. 2006, 124, 074104.

13. Jacquemin, D.; Perpete, E. A. The $\mathrm{n}->$ pi* transition in nitroso compounds: A TD-DFT study. Chemical Physics Letters 2006, 420, 529-533.

14. Jacquemin, D.; Preat, J.; Wathelet, V.; Fontaine, M.; Perpete, E. A. Thioindigo dyes: Highly accurate visible spectra with TD-DFT. J. Am. Chem. Soc. 2006, 128, 2072-2083.

15. Jacquemin, D.; Preat, J.; Wathelet, V.; Perpete, E. A. Time-dependent density functional theory determination of the absorption spectra of naphthoquinones. Chem. Phys. 2006, $328,324-332$.

16. Jacquemin, D.; Perpete, E. A.; Vydrov, O. A.; Scuseria, G. E.; Adamo, C. Assessment of long-range corrected functionals performance for $\mathrm{n}->$ pi $\left(^{*}\right)$ transitions in organic dyes. $J$. Chem. Phys. 2007, 127, 094102.

17. Jacquemin, D.; Perpete, E. A.; Scalmani, G.; Frisch, M. J.; Kobayashi, R.; Adamo, C. Assessment of the efficiency of long-range corrected functionals for some properties of large compounds. J. Chem. Phys. 2007, 126, 144105.

18. Jacquemin, D.; Perpete, E. A.; Scuseria, G. E.; Ciofini, I.; Adamo, C. Extensive TD-DFT investigation of the first electronic transition in substituted azobenzenes. Chemical Physics Letters 2008, 465, 226-229.

19. Jacquemin, D.; Perpete, E. A.; Ciofini, I.; Adamo, C. Accurate Simulation of Optical Properties in Dyes. Acc. Chem. Res. 2009, 42, 326-334.

20. Jacquemin, D.; Peltier, C.; Ciofini, I. Visible spectrum of naphthazarin investigated through Time-Dependent Density Functional Theory. Chemical Physics Letters 2010, 493, 67-71.

21. Jacquemin, D.; Adamo, C. Basis set and functional effects on excited-state properties: Three bicyclic chromogens as working examples. International Journal of Quantum Chemistry 2012, 112, 2135-2141.

22. Laurent, A. D.; Jacquemin, D. TD-DFT benchmarks: A review. Int. J. Quantum Chem. 2013, 113, 2019-2039.

23. Peach, M. J. G.; Benfield, P.; Helgaker, T.; Tozer, D. J. Excitation energies in density functional theory: An evaluation and a diagnostic test. J. Chem. Phys. 2008, 128, 044118.

24. Jacquemin, D. Excited-State Dipole and Quadrupole Moments: TD-DFT versus CC2. J. Chem. Theory Comput. 2016, 12, 3993-4003.

25. Tawada, Y.; Tsuneda, T.; Yanagisawa, S.; Yanai, T.; Hirao, K. A long-range-corrected time-dependent density functional theory. J. Chem. Phys. 2004, 120, 8425-8433. 
26. Timerghazin, Q. K.; Carlson, H. J.; Liang, C.; Campbell, R. E.; Brown, A. Computational prediction of absorbance maxima for a structurally diverse series of engineered green fluorescent protein chromophores. J Phys Chem B 2008, 112, 2533-2541.

27. Caricato, M.; Trucks, G. W.; Frisch, M. J.; Wiberg, K. B. Oscillator Strength: How Does TDDFT Compare to EOM-CCSD? J. Chem. Theory Comput. 2011, 7, 456-466.

28. Platt, J. R. Classification of Spectra of Cata-Condensed Hydrocarbons. J. Chem. Phys. 1949, 17, 484-495.

29. SerranoAndres, L.; Roos, B. O. Theoretical study of the absorption and emission spectra of indole in the gas phase and in a solvent. J. Am. Chem. Soc. 1996, 118, 185-195.

30. Robinson, D.; Besley, N. A.; Lunt, E. A. M.; O'Shea, P.; Hirst, J. D. Electronic Structure of 5-Hydroxyindole: From Gas Phase to Explicit Solvation. J Phys Chem B 2009, 113, 2535-2541.

31. Robinson, D.; Besley, N. A.; O'Shea, P.; Hirst, J. D. Calculating the Fluorescence of 5Hydroxytryptophan in Proteins. J Phys Chem B 2009, 113, 14521-14528.

32. Brand, C.; Meerts, W. L.; Schmitt, M. How and Why Do Transition Dipole Moment Orientations Depend on Conformer Structure? J Phys Chem A 2011, 115, 9612-9619.

33. Becke, A. D. Density-Functional Thermochemistry .3. the Role of Exact Exchange. $J$. Chem. Phys. 1993, 98, 5648-5652.

34. Hariharan, P. C.; Pople, J. A. Influence of Polarization Functions on Molecular-Orbital Hydrogenation Energies. Theor. Chim. Acta 1973, 28, 213-222.

35. Francl, M. M.; Pietro, W. J.; Hehre, W. J.; Binkley, J. S.; Gordon, M. S.; DeFrees, D. J.; Pople, J. A. Self-Consistent Molecular-Orbital Methods .23. a Polarization-Type Basis Set for 2nd-Row Elements. J. Chem. Phys. 1982, 77, 3654-3665.

36. Christiansen, O.; Koch, H.; Jorgensen, P. Response Functions in the Cc3 Iterative Triple Excitation Model. J. Chem. Phys. 1995, 103, 7429-7441.

37. Koch, H.; Christiansen, O.; Jorgensen, P.; deMeras, A.; Helgaker, T. The CC3 model: An iterative coupled cluster approach including connected triples. J. Chem. Phys. 1997, 106, 1808-1818.

38. Hattig, C. Structure optimizations for excited states with correlated second-order methods: CC2 and ADC(2). Adv. Quantum Chem. 2005, 50, 37-60.

39. Schreiber, M.; Silva, M. R. J.; Sauer, S. P. A.; Thiel, W. Benchmarks for electronically excited states: CASPT2, CC2, CCSD, and CC3. J. Chem. Phys. 2008, 128, 134110.

40. Silva-Junior, M. R.; Schreiber, M.; Sauer, S. P. A.; Thiel, W. Benchmarks for electronically excited states: Time-dependent density functional theory and density functional theory based multireference configuration interaction. J. Chem. Phys. 2008, $129,104103$. 
41. Kannar, D.; Szalay, P. G. Benchmarking Coupled Cluster Methods on Valence Singlet Excited States. J. Chem. Theory Comput. 2014, 10, 3757-3765.

42. Budzak, S.; Scalmani, G.; Jacquemin, D. Accurate Excited-State Geometries: A CASPT2 and Coupled-Cluster Reference Database for Small Molecules. J. Chem. Theory Comput. 2017, 13, 6237-6252.

43. Jacquemin, D. What is the Key for Accurate Absorption and Emission Calculations, Energy or Geometry? J. Chem. Theory Comput. 2018, 14, 1534-1543.

44. Wormit, M.; Rehn, D. R.; Harbach, P. H. P.; Wenzel, J.; Krauter, C. M.; Epifanovsky, E.; Dreuw, A. Investigating excited electronic states using the algebraic diagrammatic construction ( ADC) approach of the polarisation propagator. Mol. Phys. 2014, 112, 774784.

45. Harbach, P. H. P.; Wormit, M.; Dreuw, A. The third-order algebraic diagrammatic construction method (ADC(3)) for the polarization propagator for closed-shell molecules: Efficient implementation and benchmarking(a)). J. Chem. Phys. 2014, 141, 064113.

46. Becke, A. D. Density-Functional Exchange-Energy Approximation with Correct Asymptotic-Behavior. Phys. Rev. A 1988, 38, 3098-3100.

47. Lee, C. T.; Yang, W. T.; Parr, R. G. Development of the Colle-Salvetti CorrelationEnergy Formula into a Functional of the Electron-Density. Phys. Rev. B 1988, 37, 785789.

48. Perdew, J. P. Density-Functional Approximation for the Correlation-Energy of the Inhomogeneous Electron-Gas. Phys. Rev. B 1986, 33, 8822-8824.

49. Zhao, Y.; Truhlar, D. G. A new local density functional for main-group thermochemistry, transition metal bonding, thermochemical kinetics, and noncovalent interactions. $J$. Chem. Phys. 2006, 125, 194101.

50. Peverati, R.; Truhlar, D. G. M11-L: A Local Density Functional That Provides Improved Accuracy for Electronic Structure Calculations in Chemistry and Physics. J. Phys. Chem. Lett. 2012, 3, 117-124.

51. Handy, N. C.; Cohen, A. J. Left-right correlation energy. Mol. Phys. 2001, 99, 403-412.

52. Perdew, J. P.; Burke, K.; Ernzerhof, M. Generalized gradient approximation made simple. Phys. Rev. Lett. 1996, 77, 3865-3868.

53. Zhao, Y.; Truhlar, D. G. The M06 suite of density functionals for main group thermochemistry, thermochemical kinetics, noncovalent interactions, excited states, and transition elements: two new functionals and systematic testing of four M06-class functionals and 12 other functionals. Theor. Chem. Acc. 2008, 120, 215-241.

54. Boese, A.; Martin, J. Development of density functionals for thermochemical kinetics. $J$. Chem. Phys. 2004, 121, 3405-3416. 
55. Yanai, T.; Tew, D. P.; Handy, N. C. A new hybrid exchange-correlation functional using the Coulomb-attenuating method (CAM-B3LYP). Chem. Phys. Lett. 2004, 393, 51-57.

56. Peverati, R.; Truhlar, D. G. Improving the Accuracy of Hybrid Meta-GGA Density Functionals by Range Separation. J. Phys. Chem. Lett. 2011, 2, 2810-2817.

57. Chai, J.; Head-Gordon, M. Systematic optimization of long-range corrected hybrid density functionals. J. Chem. Phys. 2008, 128, 084106.

58. Chai, J.; Head-Gordon, M. Long-range corrected hybrid density functionals with damped atom-atom dispersion corrections. Phys. Chem. Chem. Phys. 2008, 10, 6615-6620.

59. Hirata, S.; Head-Gordon, M. Time-dependent density functional theory within the TammDancoff approximation. Chem. Phys. Lett. 1999, 314, 291-299.

60. Shao, Y.; Gan, Z.; Epifanovsky, E.; Gilbert, A. T. B.; Wormit, M.; Kussmann, J.; Lange, A. W.; Behn, A.; Deng, J.; Feng, X.; Ghosh, D.; Goldey, M.; Horn, P. R.; Jacobson, L. D.; Kaliman, I.; Khaliullin, R. Z.; Kus, T.; Landau, A.; Liu, J.; Proynov, E. I.; Rhee, Y. M.; Richard, R. M.; Rohrdanz, M. A.; Steele, R. P.; Sundstrom, E. J.;

Woodcock,H.Lee,,III; Zimmerman, P. M.; Zuev, D.; Albrecht, B.; Alguire, E.; Austin, B.; Beran, G. J. O.; Bernard, Y. A.; Berquist, E.; Brandhorst, K.; Bravaya, K. B.; Brown, S. T.; Casanova, D.; Chang, C.; Chen, Y.; Chien, S. H.; Closser, K. D.; Crittenden, D. L.; Diedenhofen, M.; DiStasio, R. A., Jr.; Do, H.; Dutoi, A. D.; Edgar, R. G.; Fatehi, S.; Fusti-Molnar, L.; Ghysels, A.; Golubeva-Zadorozhnaya, A.; Gomes, J.; Hanson-Heine, M. W. D.; Harbach, P. H. P.; Hauser, A. W.; Hohenstein, E. G.; Holden, Z. C.; Jagau, T.; Ji, H.; Kaduk, B.; Khistyaev, K.; Kim, J.; Kim, J.; King, R. A.; Klunzinger, P.;

Kosenkov, D.; Kowalczyk, T.; Krauter, C. M.; Lao, K. U.; Laurent, A. D.; Lawler, K. V.; Levchenko, S. V.; Lin, C. Y.; Liu, F.; Livshits, E.; Lochan, R. C.; Luenser, A.; Manohar, P.; Manzer, S. F.; Mao, S.; Mardirossian, N.; Marenich, A. V.; Maurer, S. A.; Mayhall, N. J.; Neuscamman, E.; Oana, C. M.; Olivares-Amaya, R.; O'Neill, D. P.; Parkhill, J. A.; Perrine, T. M.; Peverati, R.; Prociuk, A.; Rehn, D. R.; Rosta, E.; Russ, N. J.; Sharada, S. M.; Sharma, S.; Small, D. W.; Sodt, A.; Stein, T.; Stueck, D.; Su, Y.; Thom, A. J. W.; Tsuchimochi, T.; Vanovschi, V.; Vogt, L.; Vydrov, O.; Wang, T.; Watson, M. A.; Wenzel, J.; White, A.; Williams, C. F.; Yang, J.; Yeganeh, S.; Yost, S. R.; You, Z.; Zhang, I. Y.; Zhang, X.; Zhao, Y.; Brooks, B. R.; Chan, G. K. L.; Chipman, D. M.; Cramer, C. J.; Goddard,William A.,,III; Gordon, M. S.; Hehre, W. J.; Klamt, A.; Schaefer,Henry F.,,III; Schmidt, M. W.; Sherrill, C. D.; Truhlar, D. G.; Warshel, A.; Xu, X.; Aspuru-Guzik, A.; Baer, R.; Bell, A. T.; Besley, N. A.; Chai, J.; Dreuw, A.; Dunietz, B. D.; Furlani, T. R.; Gwaltney, S. R.; Hsu, C.; Jung, Y.; Kong, J.; Lambrecht, D. S.; Liang, W.; Ochsenfeld, C.; Rassolov, V. A.; Slipchenko, L. V.; Subotnik, J. E.; Van Voorhis, T.; Herbert, J. M.; Krylov, A. I.; Gill, P. M. W.; Head-Gordon, M. Advances in molecular quantum chemistry contained in the Q-Chem 4 program package. Mol. Phys. 2015, 113, 184-215.

61. Stanton, J. F.; Gauss, J.; Cheng, L.; Harding, M. E.; Matthews, D. A.; Szalay, P. G. CFOUR, Coupled-Cluster techniques for Computational Chemistry, a quantum-chemical program package. 
62. Dreuw, A.; Head-Gordon, M. Failure of time-dependent density functional theory for long-range charge-transfer excited states: The zincbacteriochlorin-bacterlochlorin and bacteriochlorophyll-spheroidene complexes. J. Am. Chem. Soc. 2004, 126, 4007-4016. 
For Table of Contents use only.
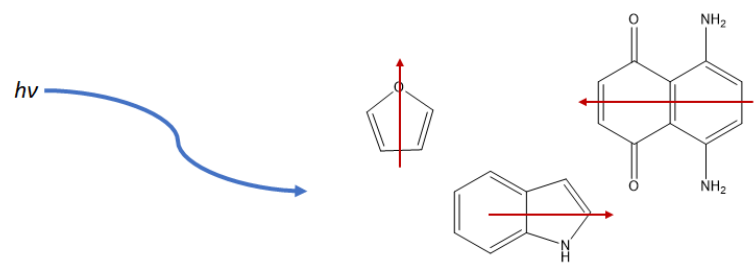

TDDFT

? 